\title{
Efficient Extraction of Structured Motifs Using Box-links
}

\author{
Alexandra M. Carvalho ${ }^{1}$, Ana T. Freitas ${ }^{1}$, Arlindo L. Oliveira ${ }^{1}$, and Marie-France \\ Sagot $^{2}$ \\ 1 IST/INESC-ID, Rua Alves Redol, 9, 1000-029 Lisboa, Portugal \\ \{asmc, atf, aml\}@algos.inesc-id.pt \\ 2 Inria Rhône-Alpes, Université Claude Bernard, Lyon I, 43 Bd du 11 Novembre 1918, 69622 \\ Villeurbanne Cedex, France \\ Marie-France.Sagoteinria.fr
}

\begin{abstract}
In this paper we propose a new data structure for the efficient extraction of structured motifs from DNA sequences. A structured motif is defined as a collection of highly conserved motifs with pre-specified sizes and spacings between them. The new data structure, called box-link, stores the information on how to jump over the spacings which separate each motif in a structured motif. A factor tree, a variation of a suffix tree, endowed with box-links provide the means for the efficient extraction of structured motifs.
\end{abstract}

Structured motifs try to capture highly conserved complex regions in a set of DNA sequences which, in the case of sequences from co-regulated genes, model functional combinations of transcription factor binding sites $[1,2,3]$. Formally, a motif is a nonempty string over an alphabet $\Sigma$ (e.g., $\Sigma=\{$ A,C,T,G $\}$ for DNA sequences). A structured motif [1] is a pair $(m, d)$ where $m$ is a $p$-tuple of motifs $\left(m_{i}\right)_{1 \leq i \leq p}$, denoting $p$ boxes, and $d$ is a $(p-1)$-tuple of pairs $\left(d_{\min _{i}}, d_{\max _{i}}\right)_{1 \leq i<p}$, denoting $p-1$ intervals of distance. In the following, we consider that all $p$ boxes of a structured motif have a fixed length $k$ and a fixed distance between boxes $d$. The general case was studied but is out of the scope of this abstract. Algorithms and complexity results are easily adaptable to the more general case.

A factor tree, also called a $k$-factor tree [4], is a data structure that indexes the factors of a string whose length does not exceed $k$. In the following we define boxlinks, whose purpose is to store the information needed to jump from box to box in a structured motif, over a factor tree. Formally, let $L$ be the set of leaves at depth $k$ of a $k$-factor tree $\mathcal{T}$ for a string $s$ of length $n$ and $L_{k}^{i}$ denote all possible $i$-tuples over $L$. A box-link of size $i$, with $1 \leq i<p$, is a $(i+1)$-tuple in $L^{i+1}$ such that there is a substring $s^{\prime}$ of $s$ where: (i) the length of $s^{\prime}$ is $i k+(i-1) d$; (ii) the $k$-length substring of $s^{\prime}$ ending at position $j k+(j-1) d$, with $1 \leq j \leq i$, is the path in $\mathcal{T}$ spelled from the root to the $j$-th leaf of the box-link tuple. Box-links can be used to extract structured motifs when built over a generalized factor tree (a factor tree for a set of $N$ sequences). However, in this case, box-links have to be endowed with a Colors Boolean array [1] in order to distinguish in which of the $N$ input sequences the corresponding boxes are linked.

In the following, we present an algorithm to build box-links. The algorithm makes use of two variables. First, the variable list $_{\text {leaf }}$ has the list of all leaves inserted in the 
factor tree, which can be easily obtained during the factor tree construction. In fact, for the sake of exposition, list leaf $_{\text {can }}$ be seen as a family of variables $\left(\text { list }_{\text {leaf }}\right)_{1 \leq i \leq N}$, where each list $_{\text {leaf }}$ i has average length $n$, the average length of an input sequence. Observe that the substring labeling the path from the root to the $j$-th leaf of list $_{\text {leaf }} f_{i}$ corresponds to the $j$-th at most $k$-length substring of the $i$-th input string. Second, the variable $b_{j}$ stores the $j$-size box-links being built. We now describe AddBoxLink function. AddBoxLink $(b, v, i)$ adds a box-link between an existing $(j-1)$-size box-link $b$ and a leaf $v$ for the $i$-th input sequence. However, it only creates a new box-link if there is not already a box-link between box-link $b$ and node $v$. In either way, creating or not a new box-link, the AddBoxLink function sets the Boolean array entry $i$ to 1 . The pseudo-code of the algorithm to build box-links is presented in Algorithm 1.

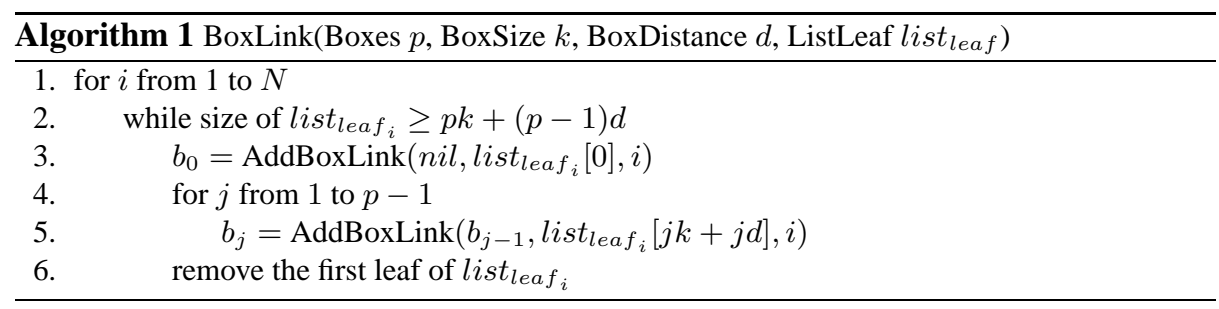

Next, we establish the complexity for Algorithm 1. Let $n_{l}$ be the number of nodes at depth $l$ of the generalized suffix tree for the same input sequences as the factor tree where the box-links are being constructed, and $b_{p}(k, d)=\min \left\{n_{k}^{p}, n_{p k+(p-1) d}\right\}$.

Proposition 1. Algorithm 1 takes $O\left(N^{2} n p\right)$ time and $O\left(N b_{p}(k, d)\right)$ space.

Proof. Step 1, 2 and 4 require $O(N), O(n)$ and $O(p)$ time, respectively. Step 5 requires $O(N)$ time, which corresponds to the creation or update of Colors array. Hence, Algorithm 1 takes $O\left(N^{2} n p\right)$ time. The space complexity is given by the number of box-links, which can be upper bounded by $b_{p}(k, d)$, times its size, which is $N$.

The use of box-links achieves a time and space exponential gain, in the worst case analysis, over approaches in [1]. Time improvement is obtained because the information required to jump from box to box in a structured motif is memorized and accessed very rapidly with box-links. Moreover, it is only required to build a $k$-factor tree, instead of a full suffix tree, or a $p k+(p-1) d$-factor tree, which leads to important space savings.

\section{References}

1. Marsan, L., Sagot, M.F.: Algorithms for extracting structured motifs using a suffix tree with an application to promoter and regulatory site consensus identification. J. Comp. Bio. 7 (2000) 345-362

2. Sharan, R., Ovcharenko, I., Ben-Hur, A., Karp, R.M.: Creme: a framework for identifying cis-regulatory modules in human-mouse conserved segments. Bioinformatics 19 (2003) i283i291

3. Segal, E., Barash, Y., Simon, I., Friedman, N., Koller, D.: A discriminative model for identifying spatial cis-regulatory modules. In: Proc. RECOMB'04. (2004) 141-149

4. Allali, J., Sagot, M.F.: The at most k-deep factor tree. Submitted for publication (2003) 